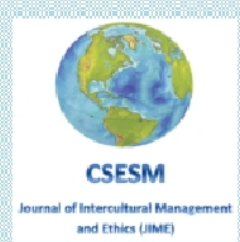

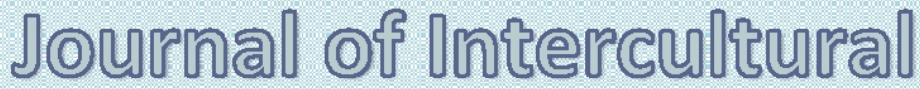

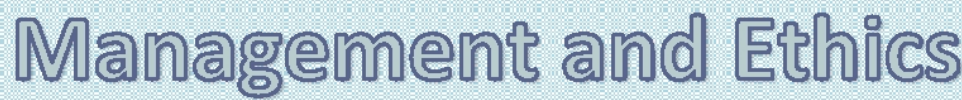

\author{
IOME
}

ISSN 2601 - 5749, ISSN-L 2601 - 5749

\section{published by zy \\ Center for Socio-Economic Studies and Multiculturalism \\ lasi, Romania \\ Waw csesmorg}




\section{TABLE OF CONTENT}

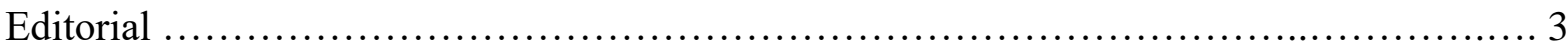

Liviu Warter

Ethics \& Consciousness in Organizations: A Conceptual Hierarchical Model

.5

Hershey H. Friedman, Kenneth A. Globerman

Ethical Challenges in Online Research.

Bianca Hanganu, Irina Smaranda Manoilescu, Beatrice Gabriela Ioan

Ethical Challenges of Digital Technologies in Covid-19 Pandemic Management. .25

Simina Petra Simion; Harald Jung

The Image of the Male and Female Doctors in the Covid-19 Pandemic. The First Pandemic with Woman Doctors in the Front Line .39 Orsolya Horber, Karoly Zilahi

Why Nations Collapse: The Rise and Fall of The United States 45

Hershey H. Friedman, Sarah Hertz

Egocentric Perceptions and Self-Serving Bias in Negotiations: Fairness, Dynamics, and Ethics 61

Oleg Komlik 


\title{
ETHICAL CHALLENGES IN ONLINE RESEARCH
}

\author{
Bianca Hanganu $^{1}$, Irina Smaranda Manoilescu ${ }^{1 *}$, Beatrice Gabriela Ioan ${ }^{1}$ \\ ${ }^{1}$ Grigore T. Popa University of Medicine and Pharmacy, Iasi, Romania \\ *Corresponding author, E-mail: manoilescuirina@yahoo.com
}

\begin{abstract}
The Internet has become an increasingly used tool in research, and the COVID-19 pandemic has increased its applicability. Researchers rely in their studies on the many benefits of the Internet- saving resources (e.g. time, money, energy), rapid dissemination to a large number of people, indefinite storage of data collected in the virtual environment. However, the use of this tool in research brings with it new challenges. The authors conducted an analysis of data published in the literature to identify the ethical challenges associated with the use of the Internet in different fields of research and the ways in which they could be overcome. The Internet can be used in any of the stages of a research: from the dissemination of the announcement, to the recruitment of participants and to the collection of data, including the dissemination of the results in specialized publications available in electronic format. The Internet generates ethical challenges in all areas of research in which it is used and which have increasingly broad boundaries: social sciences and humanities, arts, medicine, engineering. The main ethical challenges mentioned in the literature refer to respecting the autonomy of research participants (privacy, confidentiality, informed consent), risk-benefit balance (maximizing benefits and minimizing social, physical or psychological risks for participants) and justice (transparency, honesty towards participants). Online research brings both advantages and challenges; the acknowledgment and the prevention of the latter create the premises for conducting research in the appropriate ethical framework, in safe conditions for all participants.
\end{abstract}

Keywords: online research, ethical challenges, COVID-19 pandemic

\section{Introduction}

The Internet has become an increasingly used tool, its accessibility growing constantly, in multiple regions of the world, among different categories of people both in terms of age and field of activity (Roser, Ritchie \& Ortiz-Ospina, 2015). With regard to age, there is a slight tilt in the balance of frequency of Internet use among adolescents compared to the older population, with some authors even coining the term "digital natives" in reference to adolescents (Hokke et al., 2018). According to data provided by the Pew Research Center, 95\% of American teens aged between 13 and 17 are active on social media platforms, and among American adults between the ages of 30 and 49, the percentage is $82 \%$ (Anderson \& Jingjing, 2018; Pew Research Center, 2019). In the European Union, internet access increased by 26 percent between 2009 and 2019, reaching up to $90 \%$ by the end of this period (Eurostat, 2020); around the world, the number of internet users increased from $0.4 \%$ of the total population in 1996, to $49.5 \%$ of the total population in 2016 (Gupta, 2017).

The advantages offered by the Internet have found their applicability in research as well (Gupta 2017; Saberi, 2020). Researchers can rely in their studies on the use of the Internet in any of the research stages, from advertisement to publication of results (Gupta, 2017). Moreover, at present, there is the possibility to carry out studies with the most varied methodologies. Although in the past the Internet has mainly facilitated survey studies, the enablement of interpersonal communication through mobile applications and social platforms, blogs or chat rooms, nowadays researchers can conduct interviews and 
observational or interactive studies, as well as studies that analyze the content posted by users in online environment (Gupta, 2017).

The Association of Internet Researchers places online research in the following parameters of internet use: "to collect data or information; to study the manner in which people use the internet; to use and engage in data processing, analysis, or storage of datasets, databanks, and/or repositories available via the internet; to study software, code and internet technologies; to examine the design or structures of systems, interfaces, pages, and elements; to employ visual and textual analysis, semiotic analysis, content analysis, or other methods of analysis to study the web and/or internet-facilitated images, writings, and media forms; to study large scale production, use, and regulation of the internet by governments, industries, corporations, and military forces" (Markham \& Buchanan, 2012, pp. 3).

Over the time, different classifications have been made for online research methods, one of the most comprehensive being the classification provided by Moreno et al. (2013): observational methods- which involve the analysis of public information posted by participants, the latter not being aware of research; interactive methods- which involve the interaction between the researcher and the participants, in order to obtain permission for the researcher to view their content; survey methods- which involve the use of the online environment for creating and/or distributing questionnaires to potential participants, along with an informed consent form, to collect the information and interview methods- facilitated by audio-video applications (Moreno, Gonui, Moreno \& Diekema, 2013).

Advantages of Internet use in research include resource savings (time, money, energy), rapid dissemination to a large number of people, recruitment of potential participants, indefinite storage of data collected in the virtual environment, removing geographical barriers and building global communities (Gupta, 2017). COVID-19 pandemic has increased its use, with some authors even suggesting the installation of a "pandemic of online research" (De Man, Campbell, Tabana \& Wouters, 2021).

However, the use of this tool in research brings with it new challenges, including ethical, with reference to the basic principles: autonomy, justice, beneficence and nonmaleficence, transposed into issues such as privacy, transparency, informed consent, confidentiality or security of study participants and their data (Eynon, Fry \& Schroeder, 2011; Gupta, 2017; Hokke et al., 2018; Moreno et al., 2013). The level of applicability of each ethical aspect is directly proportional to the level of interaction between the participant and the researcher (Gupta, 2017).

The aim of this paper is to identify the ethical challenges associated with the use of the Internet in different fields of research and the ways in which they could be overcome, using the data published in literature.

\section{The use of the Internet in different stages of research}

The Internet has radically changed the possibilities of inter-human communication, currently being the most used way of communication in everyday life, allowing to overcome geographical barriers. The contemporary world is characterized by greater social mobility due to professional or financial reasons. Funding for research projects is sometimes limited, with researchers having to manage their allocated amounts as carefully as possible. In this context, the research facilitated by Internet is becoming increasingly used, for the numerous advantages it offers in almost all stages of research (Hokke et al., 2018): from the dissemination of the announcement, to the recruitment of participants and to the collection of data, to the dissemination of the results in specialized publications available in electronic format (Gupta, 2017).

For example, to recruit participants, researchers can share invitations or announcements almost instantly via email or social media to a large number of potential 
participants from any region of the world. In this way, geographical barriers are overcome and important financial, time and energy resources are saved. The same online methods can be used to create strategies for retention of participants- facilitating their ongoing involvement, as well as strategies for retrieving participants who- for various reasons- were lost during follow-up (Hokke et al., 2018; Saberi, 2020). Other advantages are the possibility to identify potential participants in specific groups on social platforms (for example, groups dedicated to people suffering from certain diseases) (Hokke et al., 2018; Saberi, 2020) and recruiting people to whom physical access would be difficult due to the risk of stigma (Whitaker, Stevelink \& Fear, 2017; Yuan, Bare, Johnson \& Saberi, 2014).

\section{Ethical challenges}

The Internet generates challenges in all areas of research in which it is used, areas that have increasingly wide boundaries: social sciences and humanities, arts, medicine, engineering. The main ethical challenges raised by the online research derive from the basic principles of research ethics on human subjects in general, and refer to respecting the autonomy of research participants, pursuing benefits and reducing harm to participants, and justice in conducting research (Gupta, 2017), with the mention that there are some particular features in the online environment, to be discussed further.

Autonomy. In general, autonomy of the research participants defines their conscious and voluntary participation in research and is operationalized through the informed consent form. At the same time, it involves ensuring confidentiality by securing and anonymizing participants' data, and respecting participants' privacy and dignity (Gupta, 2017; Hokke et al., 2018). Adherence to this principle also includes the protection of persons who cannot decide for themselves. Applied in the online environment, this principle brings with it the most challenges- on the one hand related to the check of the identity of the participants, and on the other hand related to data security. Data security is sometimes more difficult to manage when compared to research by conventional methods. Therefore, additional methods of data security are required (Gupta, 2017; Shelley-Egan, 2015).

Justice. This principle implies transparency and honesty towards participants, as well as their equal treatment in all stages of the research (Kitchin, 2007). Specifically, this means revealing the identity of the researcher, explaining the methods used in research (World Medical Association, 2006), the aim, the risks and the benefits of research (Gelinas et al., 2017), avoiding unnecessary burdens on participants and avoiding discrimination (World Medical Association, 2006). This principle also involves the protection of persons who cannot decide for themselves, by avoiding exploitation for purely scientific purposes (World Medical Association, 2006). In terms of equitable access to research, inequalities in internet access make potential participants from economically disadvantaged areas to be excluded from online research (Hokke et al., 2018; Saberi, 2020). The same problem occurs in case of people with a low level of education, who cannot participate in online research due to lack of knowledge of using the Internet even if they have access to it (Hokke et al., 2018). In addition, the exclusion of these populations from online research can also be a source of bias and may limit the representativeness of the study group (Hokke et al., 2018), with the impossibility of generalizing the results (Saberi, 2020). However, some authors suggest that this issue is a methodological limitation of the study rather than an ethical concern (Hokke et al., 2018), leading to participant selection bias and consequently to sample validation issues (De Man et al., 2021).

Beneficence and non-maleficence. These principles imply the obligation to maximize the benefits and minimize the social, somatic, psychological or medical risks to participants (Kitchin, 2007). Particularly for online research, participants may be harmed by the disclosure of their identity if the necessary data security measures are not applied. Disclosure 
of any type of information provided by the participants may expose them to the risk of being stigmatized, tarnished or legally charged (Townsend \& Wallace, 2016).

Another issue that can raise challenges is the requirement of obtaining the approval of a research ethics committee before the begining of the research. In this regard, a good example is Canadian Tri-Council Policy Statement, cited by Gupta (2017), which allows waiving the ethical approval in case of "research that is non-intrusive, and does not involve direct interaction between the researcher and the participants through the internet, and the one that involves collection of online documents available in the public domain (such as images, videos, or other archival materials to which the public is given uncontrolled access, for which there is no expectation of privacy) [which] are not considered under the remit of human subject research for the purpose of ethics review" (Gupta, 2017, pp. 5). However, the problem is that there are still debates on the limit between the public and the private space (Mackenzie, 2017; Warrell \& Jacobsen, 2014).

\section{Particular ethical challenges in Internet-based research}

\section{Ethical challenges in observational or non-intrusive research methods}

Despite the fact that in research using observational methods participants are not aware of research, the way in which information taken from the online environment is obtained or stored can lead to their direct or indirect identification, which may create a risk of liability or damage to reputation (Moreno et al., 2013). Therefore, despite the public nature of the information, the privacy of the participants must be respected. To this is added the knowledge and observance of the privacy policy of the website and the various legal documents in force, either international or specific to each country (Gupta, 2017; Hokke et al., 2018; Moreno et al., 2013). Although the privacy policy of different social platforms may include the public nature of certain information, it is unlikely that users will consider the possibility that it may actually be used by someone outside, including for research purposes. Therefore, this ethical issue is also raised in the use of information available on such platforms, given that users are not really aware of this possibility (Hokke et al., 2018). Still related to the privacy policy and the terms of use of different platforms, few users actually read the information contained in these policies, tending to tick box which states "I have read, understand and accept the terms of use" almost mechanically (Hokke et al., 2018). Moreover, empirical evidence even suggested that the abovementioned quotation is one of the most often encountered lies on the internet (Obar \& Oeldorf-Hirsch, 2018)

\section{Ethical challenges associated with interactive research methods}

In research that uses interactive methods, researchers reveal their identity and research/study objectives, interacting directly with participants: either to individually request access to the group of friends- which will allow the study of non-public/private posts, or to request access in a group already created on social networks, but for which access is made with the consent of a group administrator (Kitchin, 2007; Moreno et al., 2013). In this case, however, there is unanimity on the need to obtain the approval of a research ethics committee, this type of research being a type of research on human subjects. As a result, it requires ensuring the anonymity of participants and obtaining their informed and voluntary consent and ensuring the possibility of withdrawal from the study at any time. In the context of interactive research in the online environment, withdrawal from the study can be done in two ways: either by leaving the group or by eliminating the answers provided by the participant who no longer wants to be part of the study group (Kitchin, 2007). Moreover, the appearance of the researcher in the group of friends of a participant raises privacy issues, so that some authors do not particularly agree with this solution (Hokke et al., 2018). 


\section{Ethical challenges associated with online interview or survey methods}

These two types of methods are increasingly used and are excellent for online research. The ethical issues associated with these methods are related to the mandatory obtaining of informed consent, taking all measures to ensure anonymity and maintaining the confidentiality of information obtained from participants (Keller \& Lee, 2010). The consent of the participants must be:

1) fully informed, stating as mandatory information the purpose of the study, the manner of data collection, the associated risks and benefits, the way in which the anonymity and confidentiality of the participants are ensured;

2) fully voluntary, participants having the possibility to withdraw from the study at any time, for any reason and without prejudice (Gupta, 2017).

In the online environment, the consent to participate in the study can be obtained either by email or by a phrase clearly expressed at the beginning of the study (Warrell \& Jacobsen, 2014), usually by checking a box or by pressing a specific button (Hokke et al., 2018). A potential risk in this case is the inability to check the understanding of the information or the full reading of the informative content, as well as the inability to check the authenticity of the study participant (Gupta, 2017; Hokke et al., 2018), of the mental status or age of the participants (Keller \& Lee, 2010; Hokke et al., 2018). In research on minors consent must be obtained from their parents. In online research this could be problematic in the absence of direct video or at least audio interaction (Hokke et al., 2018).

\section{Particular case: research on children}

The Internet is suitable both for research on adult human subjects and children or for research on various aspects related to the family. Research using the Internet has become increasingly used in studies targeting children, especially as the Internet has become increasingly accessible on smart mobile devices, which are widely used by adolescents (Hokke et al., 2018).

In research on children, the major challenge is to obtain parental consent in the absence of physical presence and face-to-face communication with them (Close, Smaldone, Fennoy \& Grey, 2013). Suggestions for solving this problem require the parents to be thoroughly informed, so that they can trust the researcher and guarantee the quality of the research, in terms of honesty, respect and legality (Boydell, Fergie, McDaid \& Hilton, 2014; Hokke et al., 2018). Regardless of the research method, in order to fulfill this desideratum, it is indicated the personal contact of the parents and the provision of additional sources of information regarding the study, to which the parents have access even in the absence of the researcher (Close et al., 2013). Likewise, taking into account the multitude of possibilities currently offered for distance communication via the Internet, it is recommended to interact through audio and video applications, with the recording of the study participation agreement or consent (Parsons, 2015).

There are situations in which researchers suggest waiving the parental consent: ethnographic research to study the online activity of children without control or parental knowledge; situations where the need for permission would limit participation and situations where informing parents would be detrimental to potential participants (Hokke et al., 2018).

A particular situation is created by observational studies, as there is a risk of vulnerability of participants because children who use the Internet are not aware of issues related to privacy and confidentiality when expressing themselves freely on social networks, revealing personal information without realizing that they could be used by other people for various purposes, including research. In this vein, the term "digital natives" is often complemented by the term "digital naives" (Hokke et al., 2018). 


\section{Recommendations and solutions}

In general terms, the challenges associated to online research can be overcome by ensuring transparency throughout the research, offering information to participants on the collection of their personal data and on the measures applied for safeguarding confidentiality, compliance with the legal provisions in force, guaranteeing the use of information strictly to achieve the stated purpose and the possibility to withdraw from the study at any time, without any consequences for the participant (Gupta, 2017).

When using email for announcements or for study invitations, email addresses from different databases can only be used if the subjects have given their consent for the potential research participation. Likewise, if the same email is sent to several people at the same time, it is mandatory that the email addresses are hidden so that potential participants cannot see the list of people to whom the invitation was sent (Gupta, 2017).

In terms of technical aspects, measures are needed to prevent unauthorized persons from accessing the database and the computer on which the information is stored, as well as the archive on the servers, using, for example, data encryption or Secure Socket Layer (Gupta, 2017). Sometimes, security measures are also required from participants, and it is recommended to use a password-based identification system so that other people cannot access the participant's content when accessing the computer or website. Moreover, participants should also be instructed on the need to secure information and documents on their own computers so as not to be exposed to the risk of harm. A good example is research on people who are victims of domestic violence or violence from their life partner, if the same computer is used by several people or if the passwords in the email are known to the life partner. In these cases there is a risk that the violent partner will discover a copy of the informed consent form, triggering additional violence (Ellard-Gray, Jeffrey, Choubak \& Crann, 2015).

To ensure anonymity, participants can receive a number or a code formed by numbers and letters. At the same time, this will make it possible to monitor the progress of research and maintain security (Gupta, 2017).

Regarding the age, several methods can be used to verify it. For example, both age and year of birth may be required; participants can be contacted by phone or video; researchers can check profiles on social platforms- but sometimes these methods can raise privacy issues (Gupta, 2017; Hokke et al., 2018).

For the proper management of ethical issues in online research, Bender et al. (2017) propose a framework "privacy by design", suggesting researchers to develop a strategic plan to prevent harm for participants in accordance with the magnitude and probability of risk of that harm to occur (Bender, Cyr, Arbuckle \& Ferris, 2017). The problem arises, however, with the quantification of the magnitude and the person responsible for this decision, issues which are not sufficiently clarified (Gupta, 2017). Also, there is no uniformity regarding the legal/juridical classification of the violation of privacy in relation to the information accessible in the online environment within the non-interventional studies (Moreno et al., 2013). An example of a situation that does not require the approval of a research ethics committee is observational research with the retrieval of information from social platforms where the user has the opportunity to set the public or private/limited (restricted) nature of the information posted. However, to this aspect is added the way in which the information obtained is used: i.e. the publication of texts that would lead to even indirect identification (for example, by deduction) of the author, requires his/her consent. The researcher must do everything possible to minimize this risk by avoiding exact citation (for example by paraphrasing) or by masking the author's identity as much as possible by other methods, such as anonymization (Eastham, 2011; Hokke et al., 2018). 


\section{Conclusions}

The increasing accessibility of the Internet and its use by a growing number of people, both for personal and professional purposes, both on computer and mobile devices is a real help for research. The use of the Internet in research saves many financial, time and energy resources and extends research beyond geographical boundaries. However, researchers must be aware of the particularities and ethical challenges associated with online research and comply with the legal provisions in force. Knowing these challenges and developing strategies to overcome them, will facilitate access to all the benefits that the Internet can offer for research, contributing to the conduct of research in an adequate ethical and safe framework for all participants.

\section{References}

Anderson, M., \& Jingjing, J. (2018). Teens, Social Media and Technology. Washington DC. Pew Research Center. https://www.pewresearch.org/internet/2018/05/31/teens-socialmedia-technology-2018/

Bender, J. L., Cyr, A. B., Arbuckle, L., \& Ferris, L. E. (2017). Ethics and privacy implications of using the internet and social media to recruit participants for health research: A privacyby-design framework for online recruitment. Journal of Medical Internet Research, 19(4), e104.

Boydell, N., Fergie, G., McDaid, L., \& Hilton, S. (2014). Avoiding pitfalls and realising opportunities: Reflecting on issues of sampling and recruitment for online focus groups. International Journal of Qualitative Methods, 13(1), 206-223.

Close, S., Smaldone, A., Fennoy, I., Reame, N. \& Grey, M. (2013). Using information technology and social networking for recruitment of research participants: Experience from an exploratory study of pediatric Klinefelter syndrome. J Med Internet Research, 15(3), e48.

De Man, J., Campbell, L., Tabana, H., \& Wouters, E. (2021). The pandemic online research in times of COVID-19. BMJ Open, 11, e043866.

Eastham, L. A. (2011). Research using blogs for data: Public documents or private musings? Research in Nursing and Health, 34(4), 353-361.

Ellard-Gray, A., Jeffrey, N.K., Choubak, M., \& Crann, S.E. (2015). Finding the hidden participants: solutions for recruiting hidden, hart-to-reach, and vulnerable populations. International Journal of Qualitative Methods, December, 1-10.

Eurostat. (2020). Digital economy and society statistics - households and individuals. https://ec.europa.eu/eurostat/statistics-explained/index.php?title=Digital_economy _and_society_statistics_-_households_and_individuals

Eynon, R., Schroeder, R., \& Fry, J. (2009). New techniques in online research: Challenges for research ethics. 21st Century Society, 4(2), 187-199.

Gelinas, L., Pierce, R., Winkler, S., Cohen, I. G., Lynch, H. F., \& Bierer, B. E. (2017). Using social media as a research recruitment tool: Ethical issues and recommendations. The American Journal of Bioethics, 17(3), 3-14.

Gupta, S. (2017). Ethical issues in designing internet-based research: recommendations for good practice. Journal of Research Practice, 13(2), article D1.

Hokke, S., Hackworth, N.J., Quin, N., Bennetts, S.K., Yee Win, H., Nicholson, J.M., ..., Crawford, S.B. (2018). Ethical issues in using the internet to engage participants in family and child research: a scoping review, PlosOne. 13(9), e0204572.

Keller, H. E., \& Lee, S. (2010). Ethical issues surrounding human participants research using the internet. Ethics \& Behavior, 13(3), 211-219.

Kitchin, H. A. (2007). Research ethics and the internet: Negotiating Canada's Tri- Council policy statement. Halifax \& Winnipeg, Canada: Fernwood. 
Mackenzie, J. (2017). Identifying informational norms in Mumsnet Talk: A reflexive-linguistic approach to internet research ethics. Applied Linguistics Review, 8(2-3), 293-314.

Markham, A., \& Buchanan, E. (2021). Ethical decision-making and internet research: recommendations from the AoIR Ethics Working Committee (Version 2.0). https://aoir.org/reports/ethics2.pdf

Moreno, M.A., Goniu, N., Moreno, P.S., \& Diekema, D. (2013). Ethics of social media research: common concerns and practical considerations. Cyberpsychology, Behavior, and Social Networking, 16(9), 708-713.

Obar, J.A. \& Oeldorf-Hirsch, A. (2018). The biggest lie on the internet: ignoring the privacy policies and terms of service policies of social networking services. Information, Communication \& Society. https://doi.org/10.1080/1369118X.2018.1486870

Parsons, S. (2015). The potential of digital technologies for transforming informed consent practices with children and young people in social research. Social Inclusion, 3(6):5668.

Pew Research Center (2019). Social Media Fact Sheet. https://www.pewresearch.org/internet/fact-sheet/social-media/

Roser, M., Ritchie, H., \& Ortiz-Ospina, E. (2015). Internet. https://ourworldindata.org/internet.

Saberi, P. (2020). Research in the time of coronavirus: continuing ongoing studies in the midst of the COVID-19 pandemic. AIDS and Behavior, 24, 2232-2235.

Shelley-Egan, C. (2015). Ethics assessment in different fields: Internet research ethics. https://satoriproject.eu/media/2.d.2-Internet-research-ethics.pdf

Townsend, L., \& Wallace, C. (2016). Social media research: A guide to ethics. The University of Aberdeen. https://www.gla.ac.uk/media/Media_487729_smxx.pdf

Warrell, J., \& Jacobsen, M. (2014). Internet research ethics and the policy gap for ethical practice in online research settings. Canadian Journal of Higher Education, 44(1), 22-37.

Whitaker, C., Stevelink, S., \& Fear, N. (2017). The use of facebook in recruiting participants for health research purposes a systematic review. Journal of Medical Internet Research, 19(8), e290

World Medical Association. (2006). An introduction to ethics issues and principles in research involving human and animal participants. Canterbury Christ Church University, UK. https://www.canterbury.ac.uk/asset-library/research/Governance-and-Ethics/introductionto-ethics.pdf

Yuan, P., Bare, M.G., Johnson, M.O., Saberi, P. (2014). Using online social media for recruitment of human immunodeficiency virus-positive participants: a cross-sectional survey. Journal of Medical Internet Research, 16(5), e117. 